Provided for non-commercial research and education use. Not for reproduction, distribution or commercial use.

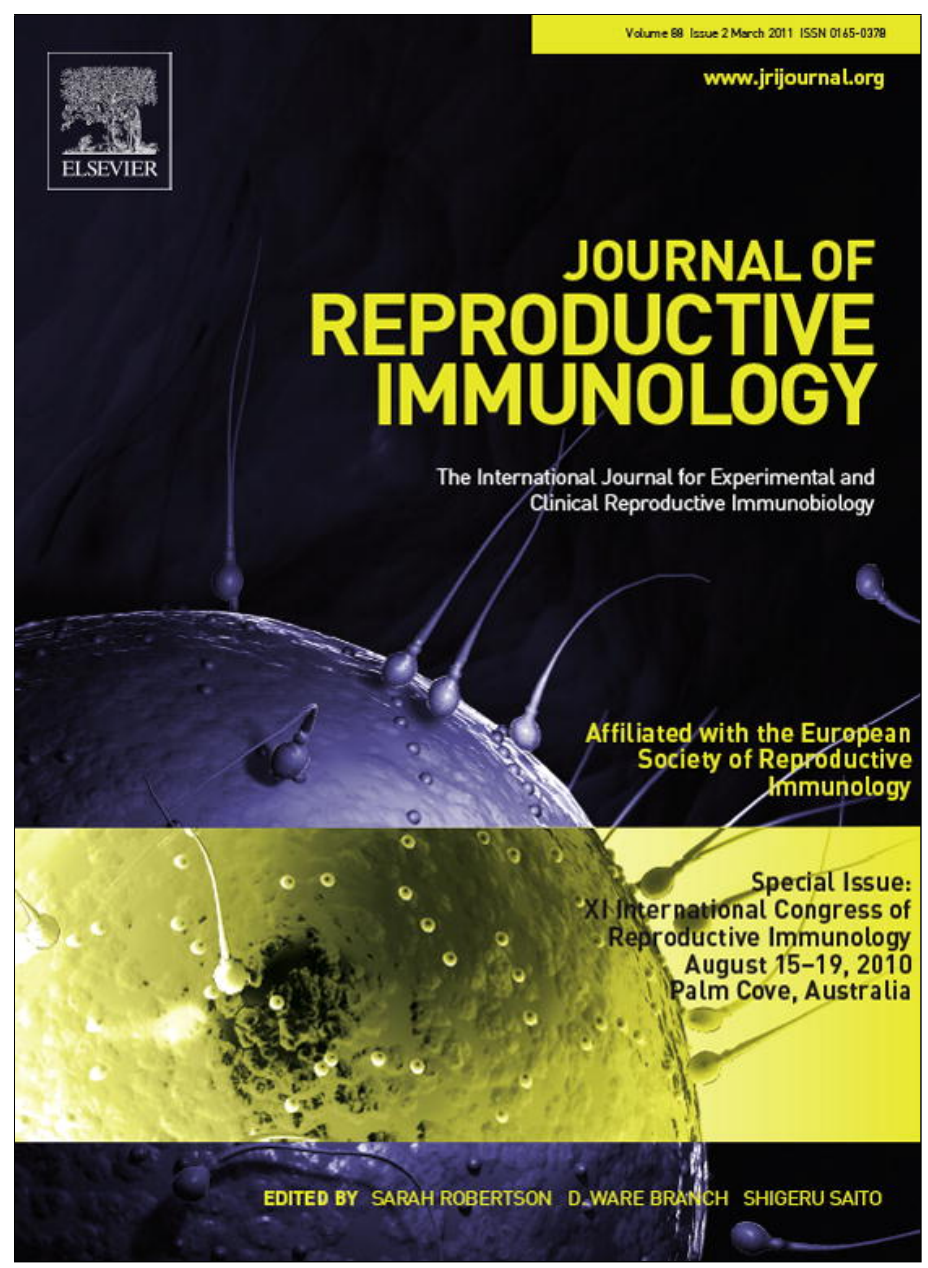

This article appeared in a journal published by Elsevier. The attached copy is furnished to the author for internal non-commercial research and education use, including for instruction at the authors institution and sharing with colleagues.

Other uses, including reproduction and distribution, or selling or licensing copies, or posting to personal, institutional or third party websites are prohibited.

In most cases authors are permitted to post their version of the article (e.g. in Word or Tex form) to their personal website or institutional repository. Authors requiring further information regarding Elsevier's archiving and manuscript policies are encouraged to visit:

http://www.elsevier.com/copyright 


\title{
New pre-conception immune biomarkers for clinical practice: interleukin-18, interleukin-15 and TWEAK on the endometrial side, G-CSF on the follicular side
}

\author{
N. Lédée ${ }^{\mathrm{a}, \mathrm{b}, *}$, M. Petitbarat ${ }^{\mathrm{a}}$, M. Rahmati $^{\mathrm{a}}, \mathrm{S}$. Dubanchet ${ }^{\mathrm{a}}$, G. Chaouat $^{\mathrm{a}}$, \\ O. Sandra ${ }^{c}$, S. Perrier-d'Hauterive ${ }^{\mathrm{b}}$, C. Munaut ${ }^{\mathrm{b}}$, J.M. Foidart ${ }^{\mathrm{b}}$ \\ a Unité 782 INSERM, Hôpital Antoine Béclère et Université Paris Sud Orsay, 32 rue des Carnets, 92141 Clamart Cedex, France \\ b University of Liege, Department of Gynaecology and Obstetrics, Centre of AMP, CHR Citadelle, B-4000 Liege, Belgium \\ c INRA/ENVA Biology of Development and Reproduction, Jouy en Josas, France
}

\section{A R T I C L E I N F O}

\section{Article history:}

Received 11 October 2010

Received in revised form 13 January 2011

Accepted 16 January 2011

\section{Keywords:}

Endometrium

Uterine receptivity

Oocyte quality

Cytokines

Embryo implantation

Uterine natural killer cells

\begin{abstract}
A B S T R A C T
Identification of biomarkers of optimal uterine receptivity to the implanting embryo as well as biomarkers of oocyte competence would undoubtedly improve the efficiency of assisted reproductive technology (ART). Expression of IL-15 and IL-18 has been shown to be different in patients with failed implantation after IVF/ICSI compared with fertile controls and both correlate with local uNK (CD56+) recruitment and angiogenesis. Tumor necrosis factor weak inducer of apoptosis (TWEAK) has been described in mice as a potent early immune regulator able to protect the conceptus. The results of our studies in human suggest that TWEAK modulates the IL-18 related cytotoxicity of uNK cells. Quantification of IL-18, TWEAK and IL-15 mRNA expression by real-time PCR in endometrial tissue collected in midluteal phase of non-conception cycles allowed documentation of physiological events that occur at the time of uterine receptivity. Such information may be useful for the physician especially in patients where embryos fail to implant. Cytokine quantification may assist in understanding the mechanisms leading to repeated IVF/ICSI failure: either depletion of cytokines necessary for the apposition-adhesion, or an excess of cytokines leading to local cytotoxicity, may impair the implantation of the embryo. Other new data suggest that a pre-conception dialogue mediated by the oocyte and the follicular fluid and the oocyte may contribute to later implantation success. Follicular concentration of G-CSF appears as a useful biomarker of oocyte competence before fertilization. Moreover both in human and animal models, evidence of a role of the endometrium as a biosensor of the embryo is emerging.
\end{abstract}

(C) 2011 Elsevier Ireland Ltd. All rights reserved.

\section{Introduction}

In the clinic, less than $5 \%$ of oocytes collected and only $20-25 \%$ of embryos transferred lead to a birth (Patrizio

\footnotetext{
* Corresponding author at: UMRS-782 INSERM université Paris XI, " implantation et dialogue cytokinique mère-conceptus " Hopital Antoine Béclère- 157, rue de la porte de Trivaux 92141 Clamart, France.. Tel.: +331453744 50; fax: +33145374450.

E-mail address: nathalie-ledee@orange.fr (N. Lédée).
}

and Sakkas, 2009). Optimisation of embryo competence as well as corresponding uterine receptivity is an absolute requirement to improve success in reproductive medicine. Such objectives rely on a better understanding of the preand peri-conception dialogue. The success of implantation depends on a receptive endometrium, a normal blastocyst and synchronized cross-talk at the maternal-fetal interface. A cascade of cytokines, chemokines and growth factors mediate this dialogue before fertilization of the oocyte and before implantation in the endometrium (Guzeloglu-Kayisli et al., 2009; Red-Horse et al., 2004; 
Salamonsen et al., 2007; van Mourik et al., 2009). To achieve the fertilization step, a good quality oocyte must meet a normal sperm with low DNA damage, leading to the development of a functionally normal blastocyst able to communicate with the maternal endometrium. The progression of pregnancy then requires immunological tolerance which allows conceptus survival. Such cross-talk involving both the immune and endocrine systems is crucial to prevent implantation failure. A better knowledge of the uterine-oocyte/embryo interaction and regulation of both the "seed and soil" during endometrial implantation is mandatory to increase the efficacy of assisted reproduction technology. Adequate coordination between embryo and mother is indeed crucial. In this short review, we will focus exclusively on some specific cytokines interleukin-18 (IL-18), IL-15 and tumor necrosis factor weak inducer of apoptosis (TWEAK) in the endometrium and granulocyte colony-stimulating factor (G-CSF, CSF3) in the follicular fluid - since they appear as potential biomarkers to document the local pre-conception environment and its equilibrium. Such immune biomarkers may be clinically useful for understanding mechanisms of implantation failure after embryo transfer.

\section{Endometrial uterine receptivity: vascular remodeling and the underlying abnormal cytokine network}

It has been proposed that uterine natural killer (uNK) cells can exert, directly or indirectly, both positive and negative control over the early events of implantation (Leonard et al., 2006; van den Heuvel et al., 2005). These cells secrete an array of cytokines important for adequate local immune regulation, angiogenesis, placental development, and establishment of pregnancy (Zhang et al., 2010). Implantation failure, recurrent miscarriage and preeclampsia have several recognized causes in common, but in most cases the precise etiology remains obscure. Various studies have identified the importance of the local immune environment and indicate the need to develop clinical tools to explore these endometrial dysregulations (Kwak-Kim and Gilman-Sachs, 2008; Quenby and Farquharson, 2006; Tuckerman et al., 2010).

Indeed the semantic distinction between implantation failure, abortion and preeclampsia might in fact be more quantitative than qualitative (Chaouat, 2008). Dysregulation of events occurring immediately after or even before conception is important determinants of embryo fate and pregnancy success. An important subset of implantation defects/early abortion cases is the consequence of a deregulation of the interleukin, tumor necrosis factor (TNF $\alpha$ ) and interferon systems, as well as networks controlled and mediated by uNK cells. Both deficient and excess expression of cytokines, as well as immune cell numbers and activation phenotype, play key roles in implantation, since these actors can have both positive and negative effects.

In our laboratory, we observed in human and mice that a proper balance in the IL-12, IL-18 and IL-15 and a correct NK activation state result in implantation and successful pregnancy. Conversely, imbalances in these parameters correlate with implantation failure or early pregnancy loss (Ledee-Bataille et al., 2005). Their expression controls the local recruitment of CD56+ uNK cells and associated sub-endometrial angiogenesis, as reflected by the endometrial vascular flow index determined by three-dimensional ultrasound (Ledee et al., 2008a,b). Such expression patterns are reproducible from one cycle to another for the same patient, and are strongly down-regulated by high concentrations of oestrogen (Ledee et al., 2006). The excellent correlation between IL-15 mRNA expression and the subendometrial vascular flow index suggests that this cytokine and the uNK cells that produce it participate in the local control of angiogenesis (Ledee et al., 2008a). Abnormal sub-endometrial vascularisation assessed by ultrasound may be the consequence of distinct cytokine dysregulation patterns. These may cause implantation failure through abnormal (insufficient or excessive) recruitment of uNK cells or through inadequate endothelial vascular remodeling before implantation.

3-D ultrasonography with vocal analysis may inform on uterine preparation for a constructive dialogue with the conceptus, and ability to provide adequate embryotrophic factors and undergo angiogenesis. However in the clinic, most ultrasonic assessments are performed either at the beginning of the cycle to evaluate the ovarian reserve or in late proliferative phase just before triggering ovulation. Much more rarely, ultrasonic assessments are performed in the mid luteal phase which is clearly the only phase relevant for this issue. Focusing on vascularisation at the time of uterine receptivity to embryo implantation is absolutely mandatory from a physiological point of view (Ledee, 2005). Patients with low sub-endometrial vascular flow index and low IL-15 mRNA are patients with insufficient uNK recruitment and/or inadequate uNK-derived angiogenic-related proteins In contrast, some patients with implantation failures exhibit very high endometrial vascular flow index and at the same time, high IL-15 and IL-18 mRNAs and $\mathrm{CD}^{56+}$ cell count. A type 1 immune response could possibly be involved in such profile of implantation failure (Kwak-Kim and Gilman-Sachs, 2008). The number of uNK cells recruited at the time of implantation as well as their state of activation need to be explored in the clinic.

In daily clinical practice, ultrasonic evaluation of subendometrial vascular flow index and measurement of the IL-15 and IL-18 mRNAs together with CD56+ uNK cell counts may thus be useful to identify those women at risk of IVF/ICSI implantation failure.

\section{The role of endometrial tumor necrosis factor-like weak inducer of apoptosis (TWEAK)}

The role of TWEAK appears very important in implantation. TWEAK is a type- 2 transmembrane protein, member of the TNF superfamily. This cytokine is described as acting in a Yin and Yang relationship with TNF $\alpha$ (Bell, 2006), because it counteracts its deleterious effects and has pro angiogenic properties (Donohue et al., 2003). Although it was first described as a weak apoptosis inducer, it triggers multiple cellular responses through its receptor, the fibroblast growth factor inducible-14 (Fn-14). These responses range from proliferation to cell death and stimulation of angiogenesis. It is highly expressed by several types of 
immune cells (such as monocytes, dendritic cells, and NK cells) and is expressed in many tissues, including the endometrium. uNK cells expressing the NKp46 cytotoxicity KIR receptor accumulate specifically in the endometrium of patients with concomitant IL-18 over-expression and low TWEAK expression. This suggests that TWEAK is involved in the control of cytotoxicity in uNK cells (Petitbarat et al., 2010).

TWEAK and its receptor Fn-14 localize in the endometrial glands and surface epithelium during the proliferative and luteal phases. They also appear scattered within the stroma, around spiral arteries, and in the luminal epithelium. No differences in immunostaining are detected among the different phases of the cycle, suggesting that TWEAK plays an early role in preventing local cytotoxicity and may counterbalance the cytotoxic function of uNK cells and favor the constructive angiogenic/immunotrophic pathways. Recent studies in humans indicate that the ratio of IL-15 and IL-18 to TWEAK mRNA expression are more important than the normalised values of IL-15, IL18 or TWEAK mRNA alone (Petitbarat et al., 2010). This testifies that the final balance of controlling signals to activation signals is likely more important than an apparently objective measure of the activation pathways. Indeed, only patients with a high IL-18/TWEAK mRNA ratio quantified by real time PCR show both excessive uNK cell recruitment with simultaneous activation of NKp46, the main endometrial activating receptor of uNK cells cytotoxicity (Petitbarat et al., 2011). In contrast, patient showing low endometrial IL-15/Fn-14 expression exhibit CD56+ uNK cell depletion. Such quantification may therefore be helpful for revealing an imbalance of crucial cytotoxic/angiogenic and immunotrophic pathways.

In summary, the ratios of IL-15/Fn14 and IL-18/TWEAK as quantified by real-time polymerase chain reaction should be evaluated prospectively as 'functional' biomarkers of uterine receptivity to document the mechanisms associated with implantation failures.

\section{IL-18/IL-15/TWEAK documentation and personalized strategies to enhance implantation}

\subsection{Patients with low endometrial angiogenesis and IL-18/TWEAK and IL-15 depletion}

In patients showing strong IL-18/TWEAK and IL-15 depletion with an absence of vascularisation, implantation failure could be related to the inability of the endometrium to react to the embryo at the time of apposition and adhesion. Hormonal administration as a consequence of ovarian hyperstimulation will increase the cytokine depletion, since endometrial exposure to a high concentration of oestrogens significantly decreases endometrial IL-18 expression (Ledee et al., 2006). A negative impact of oestrogens on IL-18 expression has been also reported in mice and pigs (Ashworth et al., 2010; Murakami et al., 2005).

In these patients, any strategies leading to the mobilization and activation of endometrial immune cells may be useful. Minimal hormonal stimulation, exposure to the male partner's seminal plasma to enhance immune cell recruitment, local endometrial injury local endometrial injury in the mild-luteal cycle preceding the IVF attempt as well as supplementation with HCG may all improves the potential for implantation. Studies on seminal plasma highlight its role in preparing the uterus for implantation by regulating recruitment and activation of $\mathrm{T}$ regulatory cells (Robertson et al., 2009). Local injury in the mid luteal phase of the cycle preceding the IVF/ICSI cycle has been reported to increase pregnancy rates (Almog et al., 2010; Barash et al., 2003; Narvekar et al., 2010). The underlying rationale is that injury causes induction of immune cells and pro-inflammatory cytokines in the endometrium (Gnainsky et al., 2010). The first known human embryoderived signal, the human chorionic gonadotropin (HCG) profoundly influences immunological tolerance and angiogenesis at the maternal-fetal interface (Perrier d'Hauterive et al., 2007). HCG intervenes in the development of local immune tolerance through apoptosis via Fas/Fas-Ligand. It modulates the Th1/Th2 balance and acts on complement $\mathrm{C} 3$ and $\mathrm{C} 4 \mathrm{~A} / \mathrm{B}$ factors influencing decidual immune function. The transient immune tolerance evident during gestation is at least partially achieved via the presence of regulatory $\mathrm{T}$ cells which are attracted by HCG into the fetal-maternal interface. HCG treatment of activated dendritic cells results in an up-regulation of MHC class II, IL-10 and indolamine 2,3 dioxygenase (IDO) expression, reducing their ability to stimulate $\mathrm{T}$ cell proliferation (Berndt et al., 2009; Tsampalas et al., 2010). Successful implantation also requires extensive endometrial angiogenesis in the implantation site. Recent data demonstrated positive angiogenic effects of HCG via its interaction with endometrial epithelial and endothelial LH/HCG receptors. Particularly, HCG induces VEGF production by endometrial epithelium, increases endothelial cell proliferation and migration of smooth muscle cells leading to the maturation of vessels, an important step for placentation (Berndt et al., 2006). HCG is also involved in the mobilisation of uNK cells via mannose receptor binding (Kane et al., 2009).

\subsection{Patients with excessive recruitment of activated $u N K$ cells}

In contrast, if patients show excessive recruitment of activated uNK cells, control of the pro-inflammatory environment is essential before embryo transfer. In such conditions, ovarian hyperstimulation as well as a strong supplementation with progesterone (for its immunosuppressive properties) in the mid luteal phase may be useful (Szekeres-Bartho, 2009; Szekeres-Bartho et al., 2009). The place of corticosteroids in such strategies is not clear, with conflicting data on actions in uNK cells. While some authors observed a decrease of uNK cells following corticosteroid administration, others authors reported the opposite. Perez et al. (2005) observed that hydrocortisone is able to enhance the recruitment of CD56 bright cells through IL-15. Corticoids were also found to promote uNK cell differentiation from CD34+ stem cells into mature CD56+ cells (Vitale et al., 2008). Drugs that inhibit the complement pathway, such as heparins as well inhibitors of ocytocin (Moraloglu et al., 2010), need to be evaluated in this context. 
To conclude, documentation of the local mobilization of uNK cells as well as their state of activation may be crucial to clinical care. Some patients with a history of embryo implantation failure show an IL-18/IL-15/uNK cell depletion and may fail at the step of embryo apposition because their endometrium is not able to adequately react to support embryo adhesion. Conversely, some other patients with the same history of implantation failure show an excessive uNK cell activation that would lead to failure of the invasion step through excessive cytotoxicity. Randomized cohort studies are now needed to demonstrate that these diagnostic tools will help the clinician in their daily practice, and such trials are mandatory in order to progress.

\section{A pre-conception dialogue: follicular G-CSF as a pre-ovulatory biomarker of oocyte competence}

Using a microbead-based multiplex sandwich immunoassay (Luminex Technology), we measured simultaneously 27 cytokines and chemokines in each follicular fluid collected from 132 individual follicles of oocytes subsequently fertilized and transferred after conventional ovarian hyperstimulation (Ledee et al., 2008 a,b). The originality of the approach was to collect individual follicular fluids and not pooled follicular fluids and to ensure traceability of each sample until birth or failure of the attempt was known. This study showed that the level of G-CSF in individual follicular fluid samples correlates with the implantation potential of the corresponding embryo. The calculation of the area under the ROC ( $\left.A U C_{R O C}\right)$ curve measures the accuracy, i.e. the ability of a factor to discriminate between two distinct outcomes. The following thresholds were used to interpret the $A_{U C} C_{\text {ROC }}$ : 0.9-1: perfect separation; 0.8-0.9: excellent discrimination; 0.7-0.8: acceptable discrimination; 0.6-0.7: poor discrimination; 0.5-0.6: no discrimination. The $A_{U C} C_{R O C}$ performance of embryo morphology as a predictor of implantation ranges between 0.65 and 0.70 for several studies (Guerif et al., 2007). In contrast, for follicular fluid G-CSF the $\mathrm{AUC}_{\mathrm{ROC}}$ distinguishing the embryos which definitely implanted from those which did not was $0.82[0.73-0.89]$ and highly significant $(p=0.0001)$. Significant differences in implantation rates were observed between the embryos with low $(<20 \mathrm{pg} / \mathrm{ml})$ and high G-CSF ( $>24 \mathrm{pg} / \mathrm{ml}$ ) (9\% versus $44 \%$ respectively, $p<0.001$ ). We subsequently reproduced the data in a cohort of 200 embryos while detailing the adequacy of distinct methods for measuring follicular fluid G-CSF (Ledee et al., 2010). Only the multiplex microbead based technology reach the required sensitivity for adequate quantification.

A third study including 83 women undergoing a modified natural IVF/ICSI cycle was subsequently conducted to provide an experimental model allowing complete traceability, whereby only one oocyte was recovered and only one embryo subsequently transferred (in contrast to the multiple embryo transfer which is conventional in France). Each follicular fluid sample was blindly tested for 26 soluble factors and each mediator evaluated as a potential biomarker of subsequent birth. Reproducibility of follicular composition was evaluated over two modified natural IVF/ICSI cycles for 15 patients. Follicular fluid
G-CSF was found to be the best predictor of subsequent birth $\left(A U C_{R O C}=0.81, p<0.0001\right)$ when using a multivariate logistic regression model (including known covariates such as age, number of IVF attempts, antral follicle count and embryo quality). The combination of follicular fluid GCSF and morphological embryo scoring on day 2 increased the $A_{U C} C_{R O C}$ to $0.86(p<0.0001)$. Birth rates per retrieval were $38 \%$ in the group with high G-CSF compared to $5 \%$ in the low G-CSF group ( $p<0.0001)$. Follicular fluid G-CSF was significantly correlated over two cycles $(r=0.71, p=0.008)$ suggesting the possible prognostic value of its documentation before starting any IVF/ICSI attempt (Ledee et al., 2011). Follicular fluid G-CSF was highly correlated with cytokines IL-7 and IL-17, suggesting key interactions within the follicle involving immune cells such as dendritic cells and regulatory $\mathrm{T}$ (Treg) cells. Thus, G-CSF in individual follicular fluids appears to correlate with the birth potential of the corresponding embryo in two distinct models of ovarian monitoring, standard ovarian hyperstimulation and modified natural IVF/ICSI cycles. This finding may have tremendous consequences for the overall morbidity related to reproductive medicine.

At the level of the reproductive tract, G-CSF has been previously shown to be secreted by granulosa cells at ovulation (Salmassi et al., 2004), then within the endometrium during the luteal phase, and finally during gestation in the placenta (Duan, 1990). More recently, a randomized controlled trial reported that GCSF administration significantly increases live-birth rates in patients with unexplained recurrent miscarriages which failed to respond to a previous immunotherapy treatment (Scarpellini and Sbracia, 2009). Almost all the miscarriages observed in our cohort were found in the group with a low follicular fluid G-CSF level. Follicular fluid G-CSF may promote local maternal-fetal tolerance (Rutella et al., 2005) or influence the oocyte's own mRNA levels or its potential for self-repair (Yannaki et al., 2005). It might also interact with cells in the local microenvironment to induce cytokines and growth factors which are necessary for embryo development and implantation.

\section{Contribution of the endometrium to the early cross-talk}

Recent studies performed in bovine models were able to demonstrate that the endometrium shows dramatic changes in gene expression related to the way the embryo was produced-by in vivo fertilization, in vitro maturation or cloning (Bauersachs et al., 2009; Mansouri-Attia et al., 2009). In addition, Mansouri-Attia et al. raised the hypothesis that the endometrium could be a natural biosensor for the embryo quality including its potential to develop to term.

In human, an in vitro confrontation model between human decidualised stromal cells and blastocyst suggested the same concept. Decidualised stromal cell gene expression showed significant variations depending on whether the blastocyst was still in development or not (Salker et al., 2010). The result of the study was somewhat surprising, given the main variation in expression indicated a control function aiming to stop the process for non-developing 
embryos and suggesting a crucial role of such regulation especially in patients with early miscarriage (Teklenburg et al., 2010). These results emphasize the importance of considering endometrial gene expression during the pre-fertilization and early pregnancy phases in order to evaluate whether the recipient tissue will respond to the embryo adequately.

\section{Conclusion}

New strategies are emerging to understand the underlying pathways leading to implantation failure and to identify specific defective pathways prior to fertilization and implantation. In this review, we focus on IL-18, IL-15 and TWEAK in the endometrium as well as G-CSF in the ovarian follicle. Identification of new biomarkers attesting to oocyte competence or adequate uterine receptivity should help us to define personalized strategies for infertility treatment. Such strategies may help to improve oocyte fertilization, embryo development and selection, uterine receptivity and ultimately endometrial-embryo cross-talk, an essential process for life.

\section{References}

Almog, B., Shalom-Paz, E., Dufort, D., Tulandi, T., 2010. Promoting implantation by local injury to the endometrium. Fertil. Steril. 94, 2026-2029.

Ashworth, M.D., Ross, J.W., Stein, D.R., White, F.J., Desilva, U.W., Geisert, R.D., 2010. Endometrial caspase 1 and interleukin-18 expression during the estrous cycle and peri-implantation period of porcine pregnancy and response to early exogenous estrogen administration. Reprod. Biol. Endocrinol. 8, 33.

Barash, A., Dekel, N., Fieldust, S., Segal, I., Schechtman, E., Granot, I., 2003. Local injury to the endometrium doubles the incidence of successful pregnancies in patients undergoing in vitro fertilization. Fertil. Steril. 79, 1317-1322.

Bauersachs, S., Ulbrich, S.E., Zakhartchenko, V., Minten, M., Reichenbach, M., Reichenbach, H.D., et al., 2009. The endometrium responds differently to cloned versus fertilized embryos. Proc. Natl. Acad. Sci. U.S.A. 106, 5681-5686.

Berndt, S., Blacher, S., Perrier d'Hauterive, S., Thiry, M., Tsampalas, M., Cruz, A., et al., 2009. Chorionic gonadotropin stimulation of angiogenesis and pericyte recruitment. J. Clin. Endocrinol. Metab. 94, 4567-4574.

Berndt, S., Perrier d'Hauterive, S., Blacher, S., Pequeux, C., Lorquet, S., Munaut, C., et al., 2006. Angiogenic activity of human chorionic gonadotropin through $\mathrm{LH}$ receptor activation on endothelial and epithelial cells of the endometrium. FASEB J. 20, 2630-2632.

Bell, E., 2006. TWEAK and TNF: Yin and Yang in innate immunity. Nat. Rev. Immunol. 6, 91-91.

Chaouat, G., 2008. Current knowledge on natural killer cells, pregnancy and pre-eclampsia. Introduction. Reprod. Biomed. Online 16, 170-172.

Donohue, P.J., Richards, C.M., Brown, S.A., Hanscom, H.N., Buschman, J., Thangada, S., et al., 2003. TWEAK is an endothelial cell growth and chemotactic factor that also potentiates FGF-2 and VEGF-A mitogenic activity. Arterioscler. Thromb. Vasc. Biol. 23, 594-600.

Duan, J.S., 1990. Production of granulocyte colony stimulating factor in decidual tissue and its significance in pregnancy. Osaka City Med. J. 36, 81-97.

Gnainsky, Y., Granot, I., Aldo, P.B., Barash, A., Or, Y., Schechtman, E., et al., 2010. Local injury of the endometrium induces an inflammatory response that promotes successful implantation. Fertil. Steril. 94, 2030-2036.

Guerif, F., Le Gouge, A., Giraudeau, B., Poindron, J., Bidault, R., Gasnier, O., et al., 2007. Limited value of morphological assessment at days 1 and 2 to predict blastocyst development potential: A prospective study based on 4042 embryos. Hum. Reprod. 22, 1973-1981.

Guzeloglu-Kayisli, O., Kayisli, U.A., Taylor, H.S., 2009. The role of growth factors and cytokines during implantation: endocrine and paracrine interactions. Semin. Reprod. Med. 27, 62-79.

Kane, N., Kelly, R., Saunders, P.T., Critchley, H.O., 2009. Proliferation of uterine natural killer cells is induced by human chorionic gonadotropin and mediated via the mannose receptor. Endocrinology 150, 2882-2888.

Kwak-Kim, J., Gilman-Sachs, A., 2008. Clinical implication of natural killer cells and reproduction. Am. J. Reprod. Immunol. 59, 388-400.

Ledee-Bataille, N., Bonnet-Chea, K., Hosny, G., Dubanchet, S., Frydman, R., Chaouat, G., 2005. Role of the endometrial tripod interleukin-18, -15, and -12 in inadequate uterine receptivity in patients with a history of repeated in vitro fertilization-embryo transfer failure. Fertil. Steril. $83,598-605$

Ledee, N., 2005. Uterine receptivity and the two and three dimensions of ultrasound. Ultrasound Obstet. Gynecol. 26, 695-698.

Ledee, N., Chaouat, G., Serazin, V., Lombroso, R., Dubanchet, S., Oger, P. et al., 2008a. Endometrial vascularity by three-dimensional power Doppler ultrasound and cytokines: a complementary approach to assess uterine receptivity. J. Reprod. Immunol. 77, 57-62.

Ledee, N., Dubanchet, S., Lombroso, R., Ville, Y., Chaouat, G., 2006. Downregulation of human endometrial IL-18 by exogenous ovarian steroids. Am. J. Reprod. Immunol. 56, 119-123.

Ledee, N., Frydman, R., Osipova, A., Taieb, J., Gallot, V., Lombardelli, L., et al., 2011. Levels of follicular G-CSF and interleukin-15 appear as noninvasive biomarkers of subsequent successful birth in modified natural in vitro fertilization/intracytoplasmic sperm injection cycles. Fertil. Steril. 95, 94-98.

Ledee, N., Lombroso, R., Lombardelli, L., Selva, J., Dubanchet, S., Chaouat G., et al., 2008b. Cytokines and chemokines in follicular fluids and potential of the corresponding embryo: the role of granulocyte colony-stimulating factor. Hum. Reprod. 23, 2001-2009.

Ledee, N., Munaut, C., Serazin, V., Perrier d'Hauterive, S., Lombardelli, L., Logiodice, F., et al., 2010. Performance evaluation of microbead and ELISA assays for follicular G-CSF: a non-invasive biomarker of oocyte developmental competence for embryo implantation. J. Reprod. Immunol. 86, 126-132.

Leonard, S., Murrant, C., Tayade, C., van den Heuvel, M., Watering, R., Croy, B.A., 2006. Mechanisms regulating immune cell contributions to spiral artery modification - facts and hypotheses - a review. Placenta 27 (Suppl. A), S40-S46.

Mansouri-Attia, N., Sandra, O., Aubert, J., Degrelle, S., Everts, R.E., GiraudDelville, C., et al., 2009. Endometrium as an early sensor of in vitro embryo manipulation technologies. Proc. Natl. Acad. Sci. U.S.A. 106, 5687-5692.

Moraloglu, O., Tonguc, E., Var, T., Zeyrek, T., Batioglu, S., 2010. Treatment with oxytocin antagonists before embryo transfer may increase implantation rates after IVF. Reprod. Biomed. Online 21, 338-343.

Murakami, Y., Otsuki, M., Kusumoto, K., Takeuchi, S., Takahashi, S., 2005. Estrogen inhibits interleukin-18 mRNA expression in the mouse uterus. J. Reprod. Dev. 51, 639-647.

Narvekar, S.A., Gupta, N., Shetty, N., Kottur, A., Srinivas, M., Rao, K.A., 2010. Does local endometrial injury in the nontransfer cycle improve the IVF-ET outcome in the subsequent cycle in patients with previous unsuccessful IVF? A randomized controlled pilot study. J. Hum. Reprod. Sci. 3, 15-19.

Patrizio, P., Sakkas, D., 2009. From oocyte to baby: a clinical evaluation of the biological efficiency of in vitro fertilization. Fertil. Steril. 91, 1061-1066.

Perez, S.A., Mahaira, L.G., Demirtzoglou, F.J., Sotiropoulou, P.A., Ioannidis, P., Iliopoulou, E.G., et al., 2005. A potential role for hydrocortisone in the positive regulation of IL-15-activated NK-cell proliferation and survival. Blood 106, 158-166.

Perrier d'Hauterive, S., Berndt, S., Tsampalas, M., Charlet-Renard, C., Dubois, M., Bourgain, C., et al., 2007. Dialogue between blastocyst hCG and endometrial LH/hCG receptor: which role in implantation? Gynecol. Obstet. Invest. 64, 156-160.

Petitbarat, M., Serazin, V., Dubanchet, S., Wayner, R., de Mazancourt, P., Chaouat, G., et al., 2010. Tumor necrosis factor-like weak inducer of apoptosis (TWEAK)/fibroblast growth factor inducible-14 might regulate the effects of interleukin 18 and 15 in the human endometrium. Fertil. Steril. 94, 1141-1143.

Petitbarat, M., Rahmati, M., Sérazin, V., Dubanchet, S., Morvan, C., Wainer, R., et al., 2011. TWEAK appears as a modulator of endometrial IL-18 related cytotoxic activity of uterine natural killers. PLoS One 6 (1), e14497.

Quenby, S., Farquharson, R., 2006. Uterine natural killer cells, implantation failure and recurrent miscarriage. Reprod. Biomed. Online 13, 24-28.

Red-Horse, K., Drake, P.M., Fisher, S.J., 2004. Human pregnancy: the role of chemokine networks at the fetal-maternal interface. Expert Rev. Mol Med. 6, 1-14.

Robertson, S.A., Guerin, L.R., Bromfield, J.J., Branson, K.M., Ahlstrom, A.C., Care, A.S., 2009. Seminal fluid drives expansion of the CD4+ CD25+ T regulatory cell pool and induces tolerance to paternal alloantigens in mice. Biol. Reprod. 80, 1036-1045. 
Rutella, S., Zavala, F., Danese, S., Kared, H., Leone, G., 2005. Granulocyte colony-stimulating factor: a novel mediator of $\mathrm{T}$ cell tolerance. J. Immunol. 175, 7085-7091.

Salamonsen, L.A., Hannan, N.J., Dimitriadis, E., 2007. Cytokines and chemokines during human embryo implantation: roles in implantation and early placentation. Semin. Reprod. Med. 25, 437-444.

Salker, M., Teklenburg, G., Molokhia, M., Lavery, S., Trew, G., Aojanepong, T., et al., 2010. Natural selection of human embryos: impaired decidualization of endometrium disables embryo-maternal interactions and causes recurrent pregnancy loss. PLoS One 5, e10287.

Salmassi, A., Schmutzler, A.G., Huang, L., Hedderich, J., Jonat, W., Mettler, L., 2004. Detection of granulocyte colony-stimulating factor and its receptor in human follicular luteinized granulosa cells. Fertil. Steril. 81 (Suppl. 1), 786-791.

Scarpellini, F., Sbracia, M., 2009. Use of granulocyte colony-stimulating factor for the treatment of unexplained recurrent miscarriage: a randomised controlled trial. Hum. Reprod. 24 (11), 2703-2708.

Szekeres-Bartho, J., 2009. Progesterone-mediated immunomodulation in pregnancy: its relevance to leukocyte immunotherapy of recurrent miscarriage. Immunotherapy 1, 873-882.

Szekeres-Bartho, J., Halasz, M., Palkovics, T., 2009. Progesterone in pregnancy; receptor-ligand interaction and signaling pathways. J. Reprod. Immunol. 83, 60-64.

Teklenburg, G., Salker, M., Heijnen, C., Macklon, N.S., Brosens, J.J., 2010. The molecular basis of recurrent pregnancy loss: impaired natural embryo selection. Mol. Hum. Reprod..
Tsampalas, M., Gridelet, V., Berndt, S., Foidart, J.M., Geenen, V., Perrier d'Hauterive, S., 2010. Human chorionic gonadotropin: a hormone with immunological and angiogenic properties. J. Reprod. Immunol. 85, 93-98.

Tuckerman, E., Mariee, N., Prakash, A., Li, T.C., Laird, S., 2010. Uterine natural killer cells in peri-implantation endometrium from women with repeated implantation failure after IVF. J. Reprod. Immunol. 87, 60-66.

van den Heuvel, M.J., Xie, X., Tayade, C., Peralta, C., Fang, Y., Leonard, S., et al., 2005. A review of trafficking and activation of uterine natural killer cells. Am. J. Reprod. Immunol. 54, 322-331.

van Mourik, M.S., Macklon, N.S., Heijnen, C.J., 2009. Embryonic implantation: cytokines, adhesion molecules, and immune cells in establishing an implantation environment. J. Leukoc. Biol. 85, 4-19.

Vitale, C., Cottalasso, F., Montaldo, E., Moretta, L., Mingari, M.C., 2008. Methylprednisolone induces preferential and rapid differentiation of CD34+ cord blood precursors toward NK cells. Int. Immunol. 20, 565-575.

Yannaki, E., Athanasiou, E., Xagorari, A., Constantinou, V., Batsis, I., Kaloyannidis, P., et al., 2005. G-CSF-primed hematopoietic stem cells or G-CSF per se accelerate recovery and improve survival after liver injury, predominantly by promoting endogenous repair programs. Exp. Hematol. 33, 108-119.

Zhang, J., Chen, Z., Smith, G.N., Croy, B.A., 2010. Natural killer celltriggered vascular transformation: maternal care before birth? Cell. Mol. Immunol. 8, 1-11. 